\title{
Religiöse Vielfalt in (post-)säkularer Gesellschaft. Umbrüche - Herausforderungen - Lernprozesse
}

\author{
Franz Gmainer-Pranzl*
}

\section{Zusammenfassung}

Der Weg zu einer religiös vielfältigen Gesellschaft war in Österreich langwierig und von vielen Schwierigkeiten geprägt, nicht zuletzt von bestimmten religionspolitischen Konstellationen. Die offizielle Anerkennung der Islamischen Glaubensgemeinschaft im Jahr 1979 markiert den Beginn religiöser Pluralität, die in den vergangenen Jahrzehnten noch deutlich zugenommen hat. Ob religiöse Vielfalt einen echten Impuls zur Weiterentwicklung einer offenen Gesellschaft vermitteln kann, hängt allerdings auch vom Verständnis des Begriffs „Religion“ ab, von der vorausgesetzten religionspolitischen Option sowie von konkreten religionstheologischen Modellen, die die Vielfalt von Religionen je anders bewerten. Religionen können gesellschaftliche Lernprozesse initiieren, was zum Beispiel den Umgang mit Fremden oder die Ausbildung eines sozialen Gewissens betrifft. Dieser Lernprozess bedarf allerdings politischer Unterstützung im Bereich der Bildung sowie der Inklusion.

Schlagwörter: Religion, Vielfalt, Postsäkularität, Religionspolitik, Offene Gesellschaft

\section{Plurality of religions in a (post-)secular society. Transformations - challenges - learning processes}

\section{Abstract}

For Austria it was a long and difficult way to become a religiously pluralistic society, not least because of special religious-political developments. The official recognition of the Islamic Community in 1979 is the beginning of religious plurality in Austria which increased in the last decades. If religious multiplicity can really help to develop an open society depends also on the concept of "religion" which one presumes, on the specific religiouspolitical options or on theological models which see religious plurality differently. Religions are able to initiate societal learning processes, for example concerning the exchange with strangers or social competence. However, this learning process needs political support in the field of education or inclusion.

Keywords: religion, plurality, postsecularity, religious politics, open society

${ }^{*}$ Franz Gmainer-Pranzl, Zentrum Theologie Interkulturell und Studium der Religionen, Universität Salzburg. E-Mail: franz.gmainer-pranzl@sbg.ac.at. 
Die Spannung zwischen Faktizität und Normativität, was gesellschaftliche Vielfalt betrifft, verschärft sich mit Blick auf Religionen insofern, als religiöse Pluralität nicht bloß die Präsenz unterschiedlicher Glaubensgemeinschaften besagt, sondern auch die Herausforderung, miteinander inkompatible Überzeugungen von „Heil“, „Wahrheit“ und „letzter Orientierung“ in einem gemeinsamen gesellschaftlichen Lebenskontext bestehen zu lassen (Mattes 2017). Läuft eine solche „Vielfalt“ nicht Gefahr, Parallelwelten zu fördern, in denen sich Anhänger einer bestimmten Religion nicht (mehr) an den Grundkonsens der Verfassung, einer demokratischen Gesellschaft, einer säkularen Grundordnung gebunden fühlen? Kommt nicht letztlich die Forderung nach Anerkennung von Pluralität denen zugute, die eine solche Vielfalt ablehnen und mit anders- oder nichtglaubenden Mitgliedern der Gesellschaft nichts zu tun haben wollen?

Dieser Beitrag geht von der Überzeugung aus, dass religiöse Vielfalt - trotz möglicher Tendenzen zu einem Separatismus oder auch Fundamentalismus - eine Chance bieten kann. Meine These lautet: Religiöse Vielfalt kann dann zu einer Inspiration für gesellschaftliche Entwicklungen werden, wenn VertreterInnen unterschiedlicher Religionsbekenntnisse sowie Menschen ohne religiöse Überzeugung bereit sind, in einen (selbst-) kritischen, wechselseitigen Lernprozess einzutreten. Zur Erläuterung dieser These (1) gehe ich zuerst auf jene Entwicklungen ein, die im 20. Jahrhundert in Österreich zu einer Herausbildung religiöser Vielfalt geführt haben, (2) schlage einige Klärungen zum Religionsbegriff sowie zum Verhältnis von Religion und Gesellschaft bzw. zu religionstheologischen Modellen vor und (3) zeige an vier Beispielen, inwiefern Religionen kreative und kritische Anstöße für eine offene, solidarische und gerechte Gesellschaft geben können. Abschließend (4) versuche ich, Möglichkeiten einer politischen Förderung und Gestaltung interreligiöser bzw. religiös-säkularer Lernprozesse aufzuzeigen bzw. auf die Chancen hinzuweisen, die in solchen Lernprozessen liegen.

\section{Umbrüche: der schwierige Weg zu religiöser Vielfalt in der österreichischen Gesellschaft}

Die gegenwärtige Vielfalt an religiösen Bekenntnissen in Österreich weist noch keine lange Geschichte auf (Zulehner 2005). Bis weit ins 20. Jahrhundert hinein stand Österreich unter dem Einfluss der römischkatholischen Kirche, auch wenn auf dem Gebiet der österreichisch-ungarischen Monarchie immer auch andere Religionsgemeinschaften wie VertreterInnen des Judentums (Keil 2015), des Islams oder des protestantischen und orthodoxen Christentums präsent waren. ${ }^{1}$ Es gab also zu keiner Zeit - nicht einmal während der Phase der Gegenreformation ${ }^{2}$ oder in den Jahren des Ständestaates - eine „rein katholische“ Gesellschaft in Österreich, aber das öffentliche Leben, die Mentalität und Kultur waren von einer Art „katholischer civil religion“ geprägt. ${ }^{3}$ Diese „katholische Symbolik“ diente jahrhundertelang dazu, eine „österreichische Identität“ zu repräsentieren; religiöse Alterität oder Pluralität erschien demgegenüber als Bedrohung der gesellschaftlichen Einheit. Wie in vielen anderen religionspolitischen Kontexten ging es auch den Habsburgern, wenn sie etwa die Protestanten unterdrückten oder auswiesen, ${ }^{4}$ nicht eigentlich um dogmatische Fragen, sondern um die Einheit des Reichs. In diesem Zusammenhang stellte das Toleranzpatent (1781) Josephs II., das den Lutheranern, Calvinisten und Griechisch-Orthodoxen eine gewisse Gleichheit mit der katholischen Mehrheit (wenn auch noch keine vollständige Gleichberechtigung) sowie Kultusfreiheit gewährte, einen ersten Schritt zur Anerkennung religiöser Diversität dar. Die Säkularisation (mit dem „Reichsdeputationshauptschluss“ von 1803) hingegen, die zu einer gewaltigen Enteignung kirchlicher Besitztümer führte, hatte eher das Erstarken einer katholischen Volkskirchlichkeit und damit ein neues Zusammengehörigkeitsgefühl zur Folge.

Das 19. Jahrhundert war in Österreich von einem Kampf zwischen jenen Kräften, die (im Anschluss an den Wiener Kongress 1814/15) die alte Ordnung und Vormachtstellung der Kirche wiederherstellen wollten, und den liberalen Kritikern einer Allianz zwischen

1 Eine gute Übersicht bietet Vocelka 2013.

2 Das 500-Jahr-Gedenken an den Beginn der Reformation, die traditionellerweise mit Luthers Publikation der Ablassthesen am 31.10.1517 angesetzt wird, bot einen guten Anlass, um das Phänomen des Geheimprotestantismus in Österreich wieder neu in den Blick zu nehmen.

3 Das Konzept der "Zivilreligion“" wurde vom US-amerikanischen Religionssoziologen Robert Bellah (1927-2013) entwickelt und meint die Gesamtheit von Überzeugungen, Werten, Symbolen und Ritualen, die von den Mitgliedern einer Gesellschaft geteilt werden - unabhängig von deren konfessioneller Zugehörigkeit.

4 Im Jahr 1837 erfolgte die letzte Vertreibung von Protestanten (aus dem Zillertal); 1731/32 verlor etwa das Land Salzburg durch die Ausweisung von Protestanten ein Fünftel seiner Gesamtbevölkerung (vgl. Dopsch 2001: 132-136). 
„Thron und Altar“ geprägt. Wichtige Eckdaten sind die Revolution von 1848; der Abschluss des Konkordats (1855); das Protestantenpatent (1861), das den evangelischen Kirchen volle politische Gleichberechtigung gewährte; und die Maigesetze von 1867/68, die zur Kündigung des Konkordats 1870 führten. Einen weiteren Schritt auf dem Weg zu einer religiös-pluralen Gesellschaft stellte die Annexion Bosnien-Herzegowinas durch Österreich-Ungarn dar, wodurch mehr als 600.00o Muslime Teil des Habsburgerreichs wurden. Ein eigenes Islamgesetz im Jahr 1912 trug dieser Veränderung Rechnung. ${ }^{5}$ Das Ende der österreichisch-ungarischen Monarchie im November 1918 führte nicht nur zu einer großen Reduktion des Staatsgebietes, sondern auch zu einer kulturellen und religiösen Homogenisierung. Die römisch-katholische Kirche stellte im neuen, kleinen Österreich die mit Abstand größte Religionsgemeinschaft dar (Weigl 2008). Als eigentliches Problem erwies sich aber nicht die statistische Überzahl der KatholikInnen, sondern deren nahezu exklusive Allianz mit der Christlich-Sozialen Partei, die spätestens seit 1927 einen militanten Kampf gegen die österreichische Sozialdemokratie führte, bis die Auseinandersetzungen nach den Februarkämpfen 1934 in den „Ständestaat“ führten (Weinzierl 1995). Emmerich Tálos bringt den religionspolitischen Kontext dieser Zeit auf den Punkt: „Die katholische Kirche bildete einen der zentralen Stützpfeiler des austrofaschistischen Herrschaftssystems“ (Tálos 2013: 240). Die Jahre bis 1938, in denen die Vertreter des „Ständestaates“ (als „Vaterländische Front" die Vertreter der früheren Christlich-Sozialen Partei) gemeinsam mit der katholischen Kirche ein System der weltanschaulichen Repression errichteten, gehören zu den dunklen Kapiteln der österreichischen Kirchengeschichte ${ }^{6}$ und waren nicht zuletzt ein ausschlaggebender Grund dafür, dass sich unterdrückte und inhaftierte BürgerInnen vom heraufziehenden Nationalsozialismus eine Befreiung aus ihrer Situation erhofften - eine Erwartung, die bekanntlich bald drastisch enttäuscht wurde.

Das Jahr 1945 bedeutete insofern eine dramatische Zäsur, was die religiöse Vielfalt in der (nun wieder) österreichischen Gesellschaft angeht, als der NSHolocaust - basierend auf dem latenten österreichi-

$5 \quad$ Zur Vorgeschichte vgl. Heuberger 2012; zum Inhalt des Islamgesetzes vgl. Bair 2012.

6 Vgl. die immer noch aufschlussreiche Auseinandersetzung bei Weinzierl 1983. - Zu den wenigen Anzeichen einer innerkirchlichen Kritik an der Politik des Ständestaates vgl. Jagschitz 1983: 507-508. schen Antisemitismus ${ }^{7}$ - das Judentum und viele bis 1938 blühende jüdischen Gemeinden der Israelitischen Kultusgemeinde nahezu völlig auslöschte. „1938 lebten in Österreich rund 180.00o Juden, nach 1945 zählte die Israelitische Kultusgemeinde keine 6.00o Mitglieder, die meisten davon alt, krank, schwach, psychisch zerstört" (Zulehner/Polak 2006: 19), konstatierten Paul Michael Zulehner und Regina Polak.

$\mathrm{Zu}$ Beginn der Zweiten Republik bildete „Österreich“ eine noch homogenere Gesellschaft, als dies 1918 der Fall war. Als ÖsterreicherIn nicht katholisch zu sein - oder gar Deutsch nicht als Muttersprache zu haben -, entsprach nicht dem gängigen Bild Österreichs in der Nachkriegszeit. Die katholische Kirche hatte zwar keine offizielle Entschuldigung für ihre Verwicklungen in den Austrofaschismus formuliert, aber durch das „Mariazeller Manifest“ (1952) einen deutlichen Schlussstrich unter die Vergangenheit gezogen: „Eine freie Kirche in einer freien Gesellschaft" lautete die Devise (Siebenrock 2013), mit der bereits Positionen des Zweiten Vatikanischen Konzils (1962-1965) zur Religionsfreiheit vorweggenommen sowie ein unbefangeneres Verhältnis zu einer pluralen Öffentlichkeit eingenommen wurde. „Keine Rückkehr zum Staatskirchentum vergangener Jahrhunderte [...]. Keine Rückkehr zu einem Bündnis von Thron und Altar [...]. Keine Rückkehr zum Protektorat einer Partei über die Kirche [...]" (Österreichische Bischofskonferenz 1952) lauten Kernsätze dieses Manifests.

Im Lauf der 1960er-Jahre kam es durch Anwerbeabkommen mit der Türkei und mit Jugoslawien, um sogenannte "Gastarbeiter" nach Österreich zu holen, zu einem Zuzug von MuslimInnen; 1979 konstituierte sich die Islamische Glaubensgemeinschaft in Österreich als Körperschaft öffentlichen Rechts. Durch die Präsenz von Mitarbeiterinnen und Mitarbeitern in internationalen Organisationen und Konzernen sowie durch Migration setzte eine allmähliche Pluralisierung der religiösen Landschaft Österreichs ein. Die Zahl der staatlich anerkannten Religionsgemeinschaften stieg; in den Schulen wurde der Religionsunterricht vielfältiger, wobei besonders die Anzahl der muslimischen SchülerInnen zunahm; Moscheen und Tempel wurden errichtet; und auch das Christentum wurde durch die

7 William Johnston spricht mit Wilhelm Marr von einem „wirtschaftlich begründeten Antisemitismus“ als einer „Bewegung, die sich vom früheren Judenhass durch das Fehlen jedes religiösen Eifers unterschied“" (Johnston 1974: 43). 
Präsenz orthodoxer und evangelikaler Kirchen bunter. Zugleich gab es immer mehr Menschen ohne religiöses Bekenntnis in Österreich, teils aufgrund einiger Austrittswellen aus der katholischen Kirche, teils einfach deshalb, weil Kinder in ihrer Familie ohne religiöses Bekenntnis aufwuchsen. Erst seit wenigen Jahren hat sich eine selbstreflexive religiöse Pluralität ausgebildet, also ein Bewusstsein davon, dass sich Menschen (insbesondere Mitglieder der „angestammten“ katholischen Kirche) in ihrer religiösen Praxis ausdrücklich als Teil einer vielfältigen Gesellschaft verstehen, zu der auch Menschen mit anderen religiösen Überzeugungen sowie Menschen ohne religiösen Glauben gehören. Multireligiöse Veranstaltungen sowie interreligiöse Dialoge sind inzwischen in Österreich zu einer gewissen Selbstverständlichkeit geworden.

Trotz diverser Lernerfahrungen, die mit diesem Umbruch $\mathrm{zu}$ einer religiös vielfältigen Gesellschaft verbunden sind, sind auch Krisen und Konflikte wahrzunehmen, die gegenwärtig sehr stark mit der Angst vor religiösem Extremismus, ${ }^{8}$ speziell im Islam, zusammenhängen. Im Zusammenleben von Säkularen und Religiösen werden zurzeit in Österreich vor allem vier Themenbereiche diskutiert: (a) Die Bedeutung religiöser Zeichen in der gesellschaftlichen Öffentlichkeit (so zum Beispiel das Kreuz im Klassenzimmer oder im Gerichtssaal, das Tragen von Kopftüchern usw.), (b) das Verhältnis von Religions- und Ethikunterricht in den (öffentlichen) Schulen, (c) der staatskirchenrechtliche Status der katholischen Kirche, wie er im Konkordat geregelt ist, sowie (d) das medial beherrschende Thema des islamischen Extremismus, angefangen von Kindergärten über Schulen und Moscheen bis hin zu Zusammenhängen mit terroristischen Aktivitäten. Sind also „Religionen“ - trotz aller Beteuerungen von Religionsgemeinschaften und TheologInnen - letztlich nicht doch eine gefährliche, verführerische Größe, die den gesellschaftlichen Zusammenhalt bedroht und vor allem junge Menschen fanatisieren kann? Hat die großzügige Anerkennung unterschiedlicher religiöser Überzeugungen nicht zu einer „neuen Unübersichtlichkeit“ geführt, die den besten Nährboden für into-

8 Ich folge hier dem Definitionsvorschlag von Bötticher/Mareš 2012: 51-63, dem zufolge "Radikalismus" eine Nischenexistenz bezeichnet, die eine spezielle weltanschauliche Haltung einnimmt, ohne deshalb Zwang auf andere auszuüben, „Extremismus“ den Versuch, andere aus der Perspektive einer vermeintlichen „Universalmoral“ zu unterwerfen, und „Terrorismus" die symbolische Inszenierung eines weltanschaulichen Extremismus. lerante, extremistische und autoritäre Bewegungen abgibt? Welchen Sinn und Wert hat in diesem Zusammenhang dann religiöse Vielfalt?

\section{Herausforderungen: „Religion“ im Plural - eine politische und theologische Zumutung}

\subsection{Religionswissenschaftliche Klärungen}

Bei der Debatte über religiöse Vielfalt, gesellschaftspolitische Herausforderungen und mögliche fundamentalistische Entwicklungen fällt auf, dass von allen Beteiligten offenbar vorausgesetzt wird, dass völlig klar sei, was mit „Religion(en)“ gemeint sei. Nicht selten ist die Meinung zu hören: „Eigentlich glauben ja alle an (irgend-)einen Gott" - wobei übersehen wird, dass der Glaube an einen Gott nicht Merkmal aller Religionen (wie etwa des Buddhismus) ist; zudem wird „Religion“ in Österreich überwiegend im Modus einer etablierten Kirche vorgestellt, was den Blick auf neue religiöse Bewegungen versperrt. Aus religionswissenschaftlicher Perspektive - also aus Sicht jener religionsbezogenen Wissenschaft, die sich historisch und systematisch mittels empirischer Kriterien (soziologisch, psychologisch, sozial- und kulturanthropologisch usw.) mit dem Forschungsbereich „Religion(en)“ auseinandersetzt - gibt es kaum einen ungeklärteren Begriff als „Religion“. Seit dem späten 19. Jahrhundert, als sich das Fach „Religionswissenschaften “ an verschiedenen Universitäten etablierte, wurde „Religion“ im Rahmen unterschiedlicher wissenschaftlicher Paradigmen verstanden. Diese Modelle folgten den philosophischen, anthropologischen und soziologischen Leitvorstellungen ihrer Zeit und wurden von neuen Konzepten kritisiert und abgelöst (Gmainer-Pranzl 2015: bes. 142-144).

So gingen etwa die Religionsethnologen am Ende des 19. Jahrhunderts selbstverständlich von der evolutionistischen Vorstellung aus, dass „Religion“ $\mathrm{zu}$ den „primitiven“ Kulturen früherer Gesellschaften (Asiens und Afrikas) gehöre und sich in der „höherentwickelten“ Gesellschaft Europas immer mehr säkularisieren würde. Gegenüber dieser - letztlich kolonialen - Konzeption machte das funktionalistische Verständnis von Religion deutlich, dass „Religion“ zu allen Zeiten derselben Logik folge: durch ihre Riten und Versprechen Antworten auf Bedürfnisse und Nöte der Menschen zu geben. Eine ebenso antievolutionistische, aber eher philosophisch geprägte Theorie stellt der religionsphänomenologische Ansatz dar, der in allen religiösen Erscheinungen (angefangen von der Steinzeit bis in die 
unmittelbare Gegenwart) die „Erfahrung des Heiligen“ sah. Eine ähnlich ungeschichtliche Perspektive nahm die strukturalistische Theorie ein; sie ging davon aus, dass die Beziehungen zwischen Menschen zu allen Zeiten von unsichtbaren, unveränderlichen Strukturen bestimmt seien, die vor allem in Mythen zur Geltung kämen, so einer der wichtigsten Vertreter des Strukturalismus, Claude Lévi-Strauss (1908-2009). Einen klaren Bruch mit phänomenologischen, funktionalistischen und strukturalistischen Ansätzen unternahm Clifford Geertz (1926-2006), der Religionen als Teil kultureller Systeme begriff. Die von ihm initiierte interpretative Wende begriff Religionen als Symbolsysteme, die Weltanschauungen und Ethiken zu einer überzeugenden Einheit verbinde. Religion sei nicht als Wesen, Funktion oder Struktur zu begreifen, sondern als Trägerin von Bedeutung. Geertz leitete mit seinem Verständnis von Religion als einem kulturellen System schließlich eine weitere Phase der Auseinandersetzung ein, die als „Krise der ethnologischen Repräsentation“ bekannt wurde. Die postkoloniale Kritik zeigte, inwiefern die „Beschreibung“ von „Religion“ den (Macht-)Interessen westlicher Wissenschaft entsprang, die für sich die Fähigkeit in Anspruch nahm, fremde kulturelle und religiöse Traditionen verstehen, darstellen und repräsentieren zu können. Dieser kulturwissenschaftliche, postkolonial geschärfte Blick charakterisiert die aktuelle religionswissenschaftliche Forschung, die auf den Prozess diskursiver Konstruktion von „Religion(en)“ hinweist, was etwa mit Blick auf die mediale Berichterstattung über „den Islam“ von Bedeutung ist (Hierl 2012; Amir-Moazami 2016).

Wenn nun in der Öffentlichkeit über Religionen und ihre gesellschaftliche Bedeutung diskutiert wird, geht es natürlich nicht um einen Fachdiskurs über Theorien und Modelle zum Verständnis von „Religion“; dennoch macht es einen gewaltigen Unterschied, ob Religionen als Funktion für den gesellschaftlichen Zusammenhalt, als bedeutungsgeladenes Symbolsystem oder als diskursive Größe vorausgesetzt wird. So macht etwa die Forderung, dass Religionen „Werte“ vermitteln sollen, nur im Kontext eines funktionalistischen Konzepts Sinn; oder die Forderung, jegliche Religion solle aus der Öffentlichkeit verbannt werden, erweist sich aus einer postkolonialen Perspektive als ein ebenso hegemonialer Diskurs wie das Aufoktroyieren von Religion. In einem strukturalistischen Paradigma ist religiöse Vielfalt kaum von Bedeutung, während sie auf dem Hintergrund eines interpretativen Zugangs die Divergenz und Konkurrenz unterschiedlicher Bedeu- tungssysteme besagt usw. Doch nicht nur das (Vor-) Verständnis von „Religion“ ist kritisch in den Blick zu nehmen, sondern auch das Verhältnis von Religion und Gesellschaft, das ein zentrales Forschungsfeld der Religionssoziologie darstellt (Gabriel/Höhn 2008; Gabriel et. al 2014; Willems et. al. 2013).

\subsection{Religionspolitische Optionen}

Die Diskussion, wie mit religiöser Vielfalt umgegangen werden soll und in welchem Verhältnis Religion(en) und Gesellschaft zu sehen sind, wird nicht zuletzt deshalb so kontrovers geführt, weil die jeweils vorausgesetzten religionspolitischen Optionen nicht klar sind. Mit Blick auf die verschiedensten religionspolitischen Szenarios lassen sich fünf Optionen, wie das Verhältnis von Religion und Gesellschaft gesehen werden kann, herausstellen: ${ }^{9}$ (a) Eine traditionale Option - die eigentlich gar keine Option im Sinn einer bewussten Entscheidung ist, sondern die faktische Prägung des Lebens durch eine religiöse Tradition, die nicht in Frage gestellt wird, ja nicht einmal als solche bewusst ist. Diese Form eines selbstverständlichen Ineinanders von Religion und Gesellschaft fand sich in segmentären Gesellschaften bzw. traditionellen (früher: „ethnischen“) Religionen, ist aber im Zeitalter der Globalisierung im Schwinden begriffen. (b) Eine integralistische Option, durch die eine Religion versucht, einer Gesellschaft ihre Maßstäbe, Überzeugungen und Werte aufzuzwingen, also die Gesellschaft in einen religiösen Innenraum zu „integrieren“. Vor dieser Versuchung ist keine Religion gefeit; in Vergangenheit und Gegenwart gibt es mehrere Beispiele für solche „Gottesstaaten“, in denen Politik und Religion letztlich identisch sind. (c) Eine säkularistische Option, ${ }^{10}$ die das Spiegelbild des religiösen Integralismus darstellt: sie will Religion(en) aus der Öffentlichkeit verbannen und sieht sie - meist auf dem Hintergrund eines evolutionistischen Religionsverständnisses - als Restbestand aus einer früheren, unaufgeklärten Phase der Menschheitsgeschichte an. (d) Eine separatistische Option, bei der sich eine Religionsgemeinschaft von sich aus dem gesellschaftlichen

$9 \mathrm{Zu}$ den folgenden Differenzierungen vgl. GmainerPranzl 2017.

10 Wichtig ist hier der Unterschied zu „säkular“: Während eine säkularistische Position Religion verdrängen will, meint „säkular“ eine Trennung von religiösem Bekenntnis und politischer Macht. Säkularismus ist eine Weltanschauung, Säkularität eine notwendige Voraussetzung einer (religiös) vielfältigen Gesellschaft. 
Kontext zurückzieht, weil sie entweder traditionale Lebensformen aufrechterhalten will (Beispiel: „Amish People“ in den USA), das gesellschaftliche Umfeld als „unrein“ ansieht (Beispiel: radikale islamistische Gruppierungen, die den „westlichen Lebensstil“ ablehnen), oder sich der staatlichen Kontrolle entziehen will (Beispiel: die chinesische Untergrundkirche, die nicht der staatlichen „Patriotischen Organisation“ angehören will). (e) Eine postsäkulare Option, die davon ausgeht, dass religiöse und säkulare BürgerInnen in einer pluralen, säkularen und liberalen Gesellschaft nicht nur koexistieren, sondern sich auf „reziproke Lernprozesse“ einlassen können (und müssen!), wie dies Jürgen Habermas immer wieder hervorhebt. Diese Position der Verhältnisbestimmung von Religion und Gesellschaft ist insofern bemerkenswert, als sie sowohl die Säkularisierungsprozesse der (westlichen) Gesellschaft als auch die ethischen, sozialen und kulturellen Ressourcen religiöser Traditionen ernst nimmt. Gegen die Etablierung einer säkularistischen Gesellschaftsordnung einerseits und die Diskursverweigerung der Mitglieder von Religionsgemeinschaften andererseits fordert Habermas - nicht zuletzt als Beitrag zu einer demokratischen Gesellschaft -, dass „die religiösen und die säkularen Bürger komplementäre Lernprozesse durchlaufen“ (Habermas 2005a: 146), und das heißt konkret: Nichtglaubende dürfen Glaubenden nicht von vornherein absprechen, aus ihren religiösen Traditionen heraus sinnvolle Antworten auf gesellschaftliche Fragen und Probleme finden zu können, und Glaubende müssen „kognitive Dissonanzen verarbeiten“ (Habermas 2005a: 143), also angesichts der kulturellen, wissenschaftlichen und politischen Herausforderungen der Gegenwart zu „neuen epistemischen Einstellungen“ (Habermas 2005a: 144) finden, von denen her sie ihre eigene Überlieferung rekonstruieren. „Postsäkularität“ meint nicht eine Alternative zu einem säkularen Gesellschaftsmodell, sondern dessen Weiterentwicklung im Kontext religiöser und weltanschaulicher Vielfalt; postsäkular ist eine Gesellschaft, wenn in ihr - in (paradoxer) Gleichzeitigkeit - sowohl Säkularisierungsschübe stattfinden als auch religiöse Überzeugungen weiterbestehen, ja sich mitunter verstärken (Höhn 2007).

\subsection{Religionstheologische Ansätze}

Religiöse Vielfalt ist also, wie die verschiedenen religionspolitischen Optionen deutlich machen, durchaus eine „Zumutung“ für die Gesellschaft; unterschiedliche religiöse Überzeugungen fordern Menschen mit und ohne religiöses Bekenntnis sowie den Gesetzgeber insgesamt dazu heraus, sich zu diesen Fragen zu positionieren, wenn es etwa um den Bereich der Bildung, um religiöse Symbole in der Öffentlichkeit, um das Halten religiöser Feiertage und Speisevorschriften oder um Seelsorge in Krankenhäusern und Seniorenheimen geht. Doch auch für die theologische Reflexion - also für jenen Religionsdiskurs, der aus der Partizipation an einer konkreten religiösen Praxis heraus erfolgt ist religiöse Vielfalt eine Zumutung, weil die Präsenz anderer Religionen den (explizit oder implizit erhobenen) Anspruch, der maßgebliche Heilsweg zu sein, in Frage stellt. Dieses Selbstverständnis von Religionen darf in der Debatte über religiöse Vielfalt nicht ausgeblendet werden: Religionen verstehen sich als „Heilswege“; sie zeigen einen Weg aus erfahrener Kontingenz (Leid, Krankheit, Tod, Schuld, Sinnlosigkeit usw.) zu einem gelingenden Leben (das theologisch mit den Begriffen „Heil“, „Erlösung“, „Fülle des Lebens“ usw. umschrieben wird). ${ }^{11}$ Wenn zum Beispiel BuddhistInnen, MuslimInnen, ChristInnen usw. in ihrer religiösen Überzeugung den Weg zum „Heil“ sehen, vertreten sie keine „intolerante“ Position, sondern praktizieren schlicht und einfach ihren Glauben, denn Religionen vertreten universale Heils- und Wahrheitsansprüche; sie interpretieren sich selbst und die anderen, den Menschen und seine Welt im Licht ihrer Überzeugung. Das Vertreten von Heils- und Wahrheitsansprüchen ist allerdings nicht - und darin liegt ein großes

11 Vgl. die treffende Charakterisierung bei Höhn 1983: 181, Fn. 244: „[...] in der Bestimmung ihres existenziellen Bezugsproblems stimmen alle Religionen darin überein, dass sie einen Grundzug menschlichen Daseins ausmachen, an dem sich das Unheile, Leidvolle, Fragmentarische, Vollendungsbedürftige, Unerlöste der ,conditio humana' festmachen lässt. Sie kommen auch darin überein, dass dies dem Menschen Fehlende und im Modus des Vermissens ihm Erschlossene nur in der Weise eines Überstiegs des Vorfindlichen erlangt werden kann. Alle Religionen verstehen sich als Wege, auf denen Menschen das Leiden an einem leidvollen und unerlösten Dasein überwinden können. Sie erschließen Möglichkeiten, wie Menschen mit den Widrigkeiten leben können, die ihnen das Leben schwer und unerträglich machen. Diese Möglichkeiten realisiert der religiöse Mensch, wenn er jenen Anleitungen folgt, die ihn in ein Verhältnis zu seinen Lebensverhältnissen setzen, das diese Lebensverhältnisse transzendiert. In diesem Verhältnis werden die leidvollen und unerlösten Daseinsverhältnisse zugleich als etwas ,Vorletztes' wahrgenommen und das Verwiesensein des Menschen auf eine, letzte 'Wirklichkeit thematisiert, von der her erst definitiv bestimmt werden kann, was letztlich aus und mit ihm werden kann.“ 
Missverständnis - mit dem fundamentalistischen Durchsetzen eigener Positionen gleichzusetzen, denn Glaubensüberzeugungen interpretieren die Welt und den Menschen immer im Licht ihrer „Wahrheit“. Wer entsprechend einer religiösen Tradition lebt, muss deshalb nicht alternative (säkulare oder religiöse) Überzeugungen bekämpfen - auch wenn er oder sie sie nicht für „wahr“ hält. Die Philosophin Anita Renusch hat diese spannende Herausforderung präzise benannt: „Religiöse Menschen können die eigenen religiösen Überzeugungen für wahr halten und inkompatible Überzeugungen für falsch; gleichzeitig sollten und können sie aber häufig auch anerkennen, dass auch diejenigen, die ihnen widersprechen, ein Recht auf genau diese Sichtweise haben“ (Renusch 2014: 321).

Genau hier setzt die anspruchsvolle Aufgabe der religionstheologischen Reflexion ein; sie fragt danach, welche Relevanz fremde religiöse Heils- und Wahrheitsansprüche für die eigene religiöse Überzeugung haben bzw. wie sich religiöse Vielfalt - und das heißt: voneinander abweichende Letztorientierungen, was die Frage nach dem „Heil“ betrifft - verstehen und rechtfertigen lässt. Im Unterschied zur Religionswissenschaft, die die Pluralität religiöser Traditionen und ihrer Geltungsansprüche „bloß“ konstatiert und empirisch untersucht, berührt diese Frage einen zentralen Nerv der Theologie; es geht um die Frage, was es für den Anhänger der Religion A bedeutet, dass eine Anhängerin der Religion B die Welt und den Menschen grundsätzlich anders interpretiert.

Aus der angloamerikanischen Diskussion hat sich in der zweiten Hälfte des 20. Jahrhunderts ein religionstheologisches Klassifikationssystem herausgebildet, das in Auseinandersetzung mit der Frage, in welchen Religionen „Heil“ und „Wahrheit“ (HW) gegeben seien, vier mögliche Modelle entwickelt hat (Schmidt-Leukel 2005: 31-192): (a) Atheismus: HW in keiner Religion. (b) Exklusivismus: HW in nur einer Religion. (c) Inklusivismus: $\mathrm{HW}$ in prinzipiell einer Religion, aber ansatzweise auch in anderen Religionen. (d) Pluralismus: ${ }^{12} \mathrm{HW}$ in mehr als einer Religion. Diese Modelle vertreten, und das ist wichtig, eine religionstheologische Logik; sie sind nicht als Anleitung zum praktischen Umgang mit religiöser Vielfalt zu verstehen. Nichtsdestotrotz beeinflussen solche Positionen die gesellschaftliche Auseinandersetzung; wer zum Beispiel einem exklusivistischen Modell folgt und von

12 „Pluralismus“ ist hier im normativen, nicht im deskriptiven Sinn gemeint. diesem überzeugt ist, wird sich in einer Debatte über die Errichtung von Moscheen vermutlich anders verhalten als ein/e AnhängerIn des religionstheologischen Pluralismus; und wenn es beispielsweise um die Frage des konfessionellen Religionsunterrichts in der Schule geht, wird ein/e AtheistIn mit hoher Wahrscheinlichkeit andere Präferenzen setzen als jemand, die/der entsprechend einer inklusivistischen Logik argumentiert. ${ }^{13}$ Auch wenn dieses religionstheologische Schema in den vergangenen Jahren einer gewissen Kritik unterzogen wurde - weil es stark von den Vorgaben analytischer Philosophie ausgeht, das Verständnis religiöser „Wahrheit“ überwiegend kognitiv geprägt ist und der gesellschaftliche Kontext von Religionen $\mathrm{zu}$ wenig berücksichtigt wird -, kann es für die theologische Zumutung sensibilisieren, die religiöse Vielfalt für die theologische Arbeit bedeutet. Dass diese Pluralität überhaupt Thema der (Religions-)Theologie geworden ist und zu einer Reihe neuer Ansätze geführt hat, stellt jedenfalls einen bemerkenswerten Fortschritt gegenüber den Versuchen dar, ein religiöses System mit den Mitteln politischer Macht in einer Gesellschaft zu etablieren bzw. eine bestimmte politische Einstellung mit Hilfe einer Religion durchzusetzen.

\section{Lernprozesse: Impulse für eine menschlichere Gesellschaft}

Religiöse Vielfalt stellt einen irreversiblen Zustand dar; der Wunsch, eine monoreligiöse oder religionslose Gesellschaftsordnung zu errichten, hat sich, wie die Geschichte zeigt, als Illusion erwiesen. Die österreichische Gesellschaft, die jahrhundertelang von einer Allianz zwischen katholischer Religion und staatlicher Macht geprägt war, ist erst dabei, religiöse Vielfalt als Normalität wahrzunehmen und darüber hinaus als Chance zu begreifen. ${ }^{14}$ Wenn allerdings von „Religi-

13 Allerdings werden die genannten religionstheologischen Positionen nur in den seltensten Fällen explizit vertreten; viele sind sich ihrer eigenen Position nicht bewusst oder vertreten sie inkonsequent. Die meisten DiskussionsteilnehmerInnen folgen religionstheologischen Intuitionen und Assoziationen (zum Beispiel: den eigenen Glauben „nicht zu verwässern“ oder „tolerant“ zu sein).

14 Diese Stimmungslage wurde deutlich, als im August 2017 die Studie „Demographie und Religion in Österreich" veröffentlicht wurde, die verschiedene Szenarien möglicher Zuwanderung durchspielt und einen deutlichen Rückgang der Katholiken sowie eine spürbare Zunahme von Nichtreligiösen und Muslimen prognostiziert (vgl. Goujon et. al. 2017: 7-21). 
onen im Plural“ gesprochen wird, muss bewusst sein, dass hier ein vieldeutiger und ambivalenter Begriff verwendet wird, der je nach vorausgesetztem Theorieansatz eine andere Bedeutung aufweist, wie der zweite Abschnitt dieses Beitrags zu zeigen versuchte. Ohne ein entsprechendes Problembewusstsein, wie es der interpretative turn und die postkoloniale Kritik vermitteln, verfällt die Auseinandersetzung mit religiöser Vielfalt essentialistischen oder funktionalistischen Vorannahmen, die dem Verhältnis von Religion und Gesellschaft angesichts der aktuellen sozialen Dynamiken nicht gerecht werden können. Diesen Herausforderungen (religiöser Extremismus, staatliche Unterdrückung von Religion oder politische Instrumentalisierung von Religion) lässt sich durch eine postsäkulare Verhältnisbestimmung am besten begegnen, und das heißt: durch die Bereitschaft, in einen wechselseitigen und (selbst-)kritischen Lernprozess einzutreten. Für die Theologie bedeutet das nach innen eine reflexive Verarbeitung des Anspruchs fremder religiöser Heils- und Wahrheitsansprüche („Religionstheologie“) und nach außen eine (interdisziplinäre) Auseinandersetzung mit Fragen der Gesellschaft sowie mit der Kritik, die gegenüber Religion(en) geäußert wird. Nur wenn das Verhältnis von Religion(en) und Gesellschaft als reziprok angesehen wird, kann es zu Lern- und Übersetzungsprozessen kommen. Die „Inspiration“, die religiöse Traditionen bieten können, betrifft das persönliche und gesellschaftliche Leben der Menschen. Habermas spricht in diesem Zusammenhang von „hinreichend differenzierte[n] Ausdrucksmöglichkeiten und Sensibilitäten für verfehltes Leben, für gesellschaftliche Pathologien, für das Misslingen individueller Lebensentwürfe und die Deformation entstellter Lebenszusammenhänge“ (Habermas 2005b: 115) und greift damit jenes Potenzial religiöser Praxis auf, das angesichts sozialer Konflikte, persönlicher Krisen oder gesellschaftlicher Probleme konkrete Lösungen bzw. Perspektiven einer menschlicheren Gesellschaft anbieten kann - auch wenn religiöse Traditionen selbst von Problemen, Krisen und Schuld geprägt sind. Das Aufzeigen möglicher Inspirationen und Impulse für eine humanere Gesellschaft bedeutet weder eine Verklärung bestimmter Religionen, einen exklusiven Anspruch auf Humanität noch eine einseitige Belehrung der Gesellschaft durch religiöse Überzeugungen. Vielmehr geht es darum, den Kontext religiöser Vielfalt in einer postsäkularen Gesellschaft als Chance zu sehen und mögliche Ressourcen für eine offenere, solidarischere und gerechtere Gesellschaft zu erschließen. Ich führe hier beispielhaft vier Impulse an, die für das gesellschaftliche Leben von Bedeutung sind: den Umgang mit dem Fremden, die Ausbildung eines sozialen Gewissens, die Orientierung an Universalität und die Etablierung einer Gedächtniskultur.

(a) Religionen leiten dazu an, sich fremden Ansprüchen, Erfahrungen und Überzeugungen zu stellen. In den Texten vieler Religionen spielt die Gestalt des Fremden eine wichtige Rolle; ihnen ist mit Aufmerksamkeit und Wertschätzung zu begegnen, weil sie möglicherweise etwas Wichtiges übermitteln oder in ihnen Gott/Götter selbst gegenwärtig ist/sind. Dazu kommt, dass Religionen ihren Anhängern immer wieder zumuten, sich herausfordernden, irritierenden, befremdenden Botschaften und Einsichten zu stellen. Religiöse Menschen sind gewissermaßen daran gewohnt, dem „Selbstverständlichen“ mit einer gewissen Skepsis zu begegnen und die eigene Aufmerksamkeit auf das zu richten, was „fremd“ erscheint. Diese Offenheit für das Fremde soll nicht xenophobe Tendenzen verleugnen, die in vielen Religionen immer wieder ausbrechen, aber einen Aspekt religiösen Lebens markieren, der besondere Beachtung verdient.

(b) Religionen bilden ein soziales Gewissen aus; sie weisen - mitunter sehr nachdrücklich - auf die Pflicht der Gläubigen hin, Arme zu unterstützen, bzw. schärfen das Bewusstsein für die Not anderer. Der Fastenmonat Ramadan im Islam, der die Fastenden ganz konkret erfahren lässt, wie es sich ohne Wasser und Nahrung anfühlt, ist ein markantes Beispiel für den Ernst, mit dem Religionen soziale Lebenshaltungen einüben. Natürlich gibt es auch das Gegenteil davon: religiöse Vertreter auf Seiten der Reichen und Mächtigen bzw. religiöse Bewegungen, die auf Wohlstand und politischen Einfluss aus sind wie die „Theologie der Prosperität“ . ${ }^{15}$ Dennoch: Es ist kein Zufall, dass religiöse Gruppen immer wieder zu den schärfsten Kritikern einer Anhäufung von Reichtum, einer unsozialen Gesellschaftsordnung und ausbeuterischer Lebensund Arbeitsbedingungen gehören.

(c) Religionen vertreten universale Ansprüche. Sie sehen das, was sie verkünden, nicht nur für eine bestimmte Gruppe oder ein Land als gültig an, sondern für alle Menschen. Aus dieser Orientierung an Univer-

15 Diese vor allem in Lateinamerika und Westafrika verbreitete Strömung vertritt - in deutlichem Gegensatz zur gesellschaftskritischen "Theologie der Befreiung“ - die Auffassung, individueller Wohlstand sei ein Zeichen des Segens Gottes. 
salität heraus kann eine starke Sensibilität für globale Zusammenhänge entstehen bzw. ein Bewusstsein, das die vertrauten kulturellen und gesellschaftlichen Kontexte relativiert und ein Gefühl der Zusammengehörigkeit über nationale und soziale Grenzen hinaus entwickelt. Zugleich sind Religionen aber auch selbst gefährdet, einem nationalistischen Fanatismus oder kulturellen Traditionalismus zu verfallen. Religionen können rechtspopulistischen Kulturalisierungs- und Nationalisierungstendenzen äußerst ablehnend gegenüberstehen oder sich von ihnen vereinnahmen lassen; sie können rassistische und sexistische Diskurse klar ablehnen oder selbst übernehmen. Der Impuls, die Identität des Menschlichen in einem universalen Horizont zu denken, lässt sich jedenfalls nicht mehr „einfangen“ und rückgängig machen; die „Universalität", die Religionen beanspruchen, gilt in diesem Sinn auch gegen die Religionen selbst, was sich vor allem an den Menschenrechten zeigt. ${ }^{16}$

(d) Religionen sind ein Speicher von Menschheitserfahrungen; sie bilden zum einen große Reservoirs an Erinnerungen und stiften zum anderen dazu an, Erinnerungen zu pflegen. Sie begründen in vielen Fällen eine eindrückliche Gedächtniskultur. Hier ist vor allem auf das Judentum hinzuweisen, das sowohl durch seine zentrale Gedächtnisfeier, die Pessach-Haggadah, ${ }^{17}$ als auch durch die Erfahrung des Holocaust zum religiösen und gesellschaftlichen Gedächtnisträger par excellence geworden ist. Mit „Erinnerung“ ist nicht bloß das Zurückdenken an vergangene Ereignisse gemeint, sondern die kritische Vergegenwärtigung des Verdrängten, Vergessenen und Unterdrückten in einer Gesellschaft, die von Amnesie geprägt ist (Münz 1996: 401-456). Dem „Vergessen“ schmerzlicher und schuldhafter Phasen der Geschichte gegenüber bringt die jüdische Gedächtniskultur einen unersetzlichen Beitrag zur Entwicklung einer menschlichen Gesellschaft ein, in der nicht einfach das Recht des Stärkeren gilt und Unrecht „vergessen“ wird. Dass die Erinnerung an die Opfer der Geschichte - ganz gleich, von wem sie wachgehalten wird - nicht selten als Bedrohung und Nestbeschmutzung angesehen wird, spricht umso mehr für die unverzichtbare Bedeutung einer „gefähr-

16 „Die Menschenrechte, zu denen unablösbar Gewissens- und Religionsfreiheit zählen, implizieren, dass der Wahrheitsanspruch einer Religion hinter das Recht des Menschen zurücktreten muss" (Heinzmann 2010: 93).

17 Das sind die Texte, die beim rituellen Mahl im Kreis der Familie rezitiert werden, um der Befreiung aus der Sklaverei in Ägypten zu gedenken. lichen Erinnerung "18 die wie ein Stachel im Fleisch der Gesellschaft steckt.

\section{Anstöße: zur politischen Gestaltung religiöser und weltanschaulicher Pluralität}

Die angesprochenen Lernprozesse ergeben sich nicht einfach von selbst, sondern bedürfen einer politischen Förderung und Gestaltung. Wenn etwa - um das zuletzt genannte Beispiel aufzugreifen - die Entwicklung einer Gedächtniskultur nicht gefördert, sondern vielleicht sogar unterdrückt wird, ergeben sich andere Folgen für das gesellschaftliche Leben als im Kontext einer Politik, in der den Opfern von Gewalt und Unterdrückung im öffentlichen Gedenken der gebührende Platz eingeräumt wird. Es liegt im Interesse der gesamten Gesellschaft, Lernprozesse sowohl in Religionsgemeinschaften als auch auf Seiten säkularer BürgerInnen zu fördern (Habermas 2005a), weil dadurch die Fähigkeit, Pluralität und Alterität als positive Herausforderung zu sehen, sowie eine Haltung der Anerkennung gestärkt wird. Lernprozesse im Sinn postsäkularer Reziprozität zu unterstützen, ist ein Beitrag zur (Selbst-)Ermächtigung und Emanzipation von Menschen - und somit auch ein Impuls zu einer demokratischen Entwicklung.

Ich möchte im Folgenden vier mögliche Anstöße einer politischen Förderung interreligiöser bzw. religiös-säkularer Dialog- und Lernprozesse anführen und einen bildungspolitischen, einen polylogen, einen empathischen sowie einen religionspädagogischen Impuls skizzieren.

(a) Der bildungspolitische Impuls geht weit über die Frage religiöser Pluralität hinaus. Angesichts einer starken Tendenz zu einer Ökonomisierung der Bildung ist zu fragen, ob nicht jene wissenschaftlichen Disziplinen, die sich mit kulturellen Traditionen, religiösen Überzeugungen, Fremdsprachen (auch sogenannten "toten“ Sprachen), speziellen historischen Fragestellungen usw. beschäftigen, in Zeiten gesellschaftlicher Pluralisierung gestärkt oder zumindest unterstützt werden sollten. Die Rede von den „Orchideenfächern“ suggeriert ökonomische Vernunft und Zeitgemäßheit, blendet aber die bleibende, ja sogar zunehmende Relevanz kulturund religionswissenschaftlicher, historischer und philologischer Forschung völlig aus. Wer hätte noch vor zwanzig Jahren gedacht, wie wichtig einmal Arabisch

18 Diesen Begriff brachte Johann Baptist Metz, prominenter katholischer Vertreter einer „Politischen Theologie“, in die Debatte ein (vgl. Metz 1977: 176-180). 
oder Chinesisch sein würden? Mit welchem Interesse werden gegenwärtig nicht Fragen der Koranauslegung, des Verständnisses des Buddhismus oder bestimmter biblischer Texte diskutiert? Wenn „Bildung in einem unverkürzten Sinn“, so Konrad Paul Liessmann, „von der Vergangenheit, vom Wissen, den Technologien, den Kunstwerken, den Religionen und Weltanschauungen, den Literaturen, die von Menschen hervorgebracht wurden und auf denen Menschen aufbauen und an denen sie weiterarbeiten können" (Liessmann 2017: 52-53), lebt, darf sie nicht auf „Know how“ im Dienst technischer und ökonomischer Kompetenzen reduziert werden, sondern braucht immer auch eine profunde Kenntnis jener Traditionen, aus denen heraus sich eine plurale Gesellschaft konstituiert.

(b) Ein Anstoß zu einer polylogen - das heißt: vielsprachigen, mit unterschiedlichsten Positionen kommunikationsfähigen - Lebens- und Denkform ${ }^{19}$ wird alle Bildungs- und Lernprozesse unterstützen, bei denen SchülerInnen und Studierende neuen und fremden Lebenskontexten begegnen können: globale Bildung, Antirassismus, Interesse für Fremdsprachen sowie für (bisher) unbekannte Lebensformen bzw. kulturelle, politische und religiöse Traditionen. Angesichts der gegenwärtigen „Verösterreicherung“, wie sie der Religionspädagoge Martin Jäggle in den aktuellen medialen Entwicklungen konstatiert und als „Realitätsflucht“ bezeichnet (Jäggle 2017: 3), wird deutlich, um welche politische Herausforderung es geht: Pluralität und Heterogenität nicht als „Problem“ wahrzunehmen, vor dem es sich durch Heimattümelei oder - verstärkt noch - durch identitäres Denken abzuschirmen gilt, sondern Menschen zu befähigen, sich mit (selbst-)kritischer Aufmerksamkeit in einer von Vielfalt geprägten Gesellschaft zu bewegen. Eine solche „Polylogfähigkeit“ bedeutet nicht - wie dies rechtspopulistische Strömungen suggerieren -, die eigene Herkunft und kulturelle Prägung zu verleugnen, sondern sie im Kontext eines vielfältigen Lebensumfelds neu wahrzunehmen. In diesem Sinn stellt die „Fähigkeit, das eigene Land als Teil einer komplizierten Weltordnung zu sehen" (Nussbaum 2016, 41), und nicht eine identitäre Regression auf die eigene „Identität“ die entscheidende Voraussetzung für das Bewältigen kommender Herausforderungen dar.

19 Der Begriff „Polylog“ wurde vom österreichischen Philosophen Franz Martin Wimmer entwickelt und meint die - zumindest ansatzhafte - Fähigkeit, anderen Positionen, Traditionen und Haltungen vorbehaltlos und lernbereit $\mathrm{zu}$ begegnen (vgl. Wimmer 2004: 66-73). (c) Der „empathische“ Impuls, der politisch zu setzen wäre, geht von einer Erfahrung aus, der in Gesellschaften, die von Migration, Pluralität, Exklusion und Xenophobie geprägt sind, eine meist völlig verkannte Bedeutung zukommt: die Verweigerung von Anerkennung sowie die Demütigung von Angehörigen einer kulturellen/religiösen Minderheit. Wenn Menschen, die gesellschaftliche Transformationsprozesse $\mathrm{zu}$ bewältigen haben, keine Unterstützung bzw. kein Verständnis erfahren, sind sie auch nicht in der Lage, sich auf Lernprozesse einzulassen; im Gegenteil: sie stellen sich dagegen und entwickeln mitunter massive Ressentiments - ein Problem, das Jürgen Habermas bereits früh erkannt und analysiert hat (Habermas 2005a: 142). Wer sich von gesellschaftlichen Entwicklungen ausgeschlossen fühlt, entwickelt keine Bereitschaft, Vielfalt als Wert anzuerkennen, und fühlt sich möglicherweise zu identitären Politiken hingezogen. Fehlende Anerkennung kann schließlich im ungünstigsten Fall zu einer gewaltförmigen Ablehnung von Vielfalt und zu politischem Extremismus führen, wie dies Abd Samad Moussaoui in seinem Buch über seinen Bruder Zacarias, der zum Terroristen wurde, eindrücklich schildert. Als Kind marokkanischer Eltern erfuhr Zacarias in der französischen Gesellschaft meistens nur Ablehnung, Rassismus und Verachtung, was ihn schlussendlich in die Arme einer islamistischen Terrororganisation führte. Abd Samad resümiert die Entwicklung seines Bruders: „Zacarias fühlt sich als Franzose nicht recht wohl in seiner Haut und spricht als Marokkaner nicht einmal Arabisch. Zu welcher Gemeinschaft soll er sich zählen? Seine Verlorenheit hat sicher dazu beigetragen, sein Zugehörigkeitsgefühl zu einer Gruppe zu fördern, die ihn bei sich aufgenommen hat. Die ständigen Schmähungen sämtlicher sunnitischer Lehren und Autoritäten haben ihn mir entfremdet und seine Kritikfähigkeit vollkommen abgestumpft“ (Moussaoui 2002: 143). Gewiss gibt es kein unfehlbares Rezept, wie jemand vor Extremismus bewahrt werden kann; wer sich aber als Teil einer kulturell, politisch und religiös vielfältigen Gesellschaft anerkannt erfährt, wird wohl kaum auf den Gedanken kommen, sich mit Hass und Gewalt gegen andere zu richten.

(d) Der religionspädagogische Impuls, den politische Maßnahmen setzen können, betrifft schließlich vor allem die Religionsgemeinschaften selbst: Die Standards der Vermittlung religiöser Inhalte müssen dem Problembewusstsein, der Komplexität und der politischen Reflexivität des aktuellen gesellschaftlichen Kontextes entsprechen. Zum einen muss es den 
Angehörigen einer Religionsgemeinschaft selbst ein zentrales Anliegen sein, Anschluss an den gegenwärtigen intellektuellen und kulturellen Diskussionsstand zu finden und ihre eigenen Überzeugungen angesichts der Fragen und Probleme der Gegenwart zu rekonstruieren; Religionen können nicht einfach in den „Untergrund" gehen und sich von den gesellschaftlichen Auseinandersetzungen verabschieden. Zum anderen muss aber auch die gesellschaftliche Öffentlichkeit ein Interesse daran haben, dass religiöse BürgerInnen reflexions-, diskussions- und auskunftsfähig sind. Religiöse Pluralität kann nicht einfach bedeuten, dass verschiedene Gruppen nebeneinander bestehen, in denen Menschen „an irgendetwas glauben“, sondern dass Angehörige unterschiedlicher Religionsgemeinschaften untereinander sowie mit Menschen ohne religiöse Zugehörigkeit in Dialog treten und sich mit Fragen, die alle betreffen (zum Beispiel Arbeitslosigkeit, soziale Gerechtigkeit, Bildung, Gesundheit, Migration usw.), auseinandersetzen. Dementsprechend sollte die Aus- und Weiterbildung von VertreterInnen religiöser Überzeugungen jene Kompetenzen und kritischen Potenziale vermitteln, die in einer pluralen und globalisierten Gesellschaft erforderlich sind. Das bedeutet nicht, den Religionsgemeinschaften inhaltliche Vorgaben bezüglich ihrer Glaubensüberzeugungen zu machen, sondern sie zu befähigen, in den gesellschaftlichen Diskurs einzutreten und sich als zivilgesellschaftliche Akteure zu begreifen.

Die Vielfalt von Religionen, die in ihren Wissensbeständen, Ritualen, Symbolen und ethischen Konzepten Potenziale zur Lebensgestaltung bergen, kann in einer (post-)säkularen Gesellschaft eine Chance darstellen, Menschen mit unterschiedlichen Lebenserfahrungen, Überzeugungen und Weltanschauungen ins Gespräch zu bringen und neue Einsichten zu ermöglichen. Ein solcher interreligiöser Dialog muss auch jene Menschen einbeziehen, die an keine religiöse Tradition gebunden sind, um die VertreterInnen der Religionen an ihre eigene Relativität zu erinnern und säkularen MitbürgerInnen bewusst zu machen, wie groß die religiöse und weltanschauliche Vielfalt ist, aus der Menschen Kraft für ihr gesellschaftliches Engagement schöpfen. Wenn religiöse Vielfalt nicht als Folklore inszeniert wird, sondern als Konsequenz einer offenen Gesellschaft gelebt wird, in der die Suche nach tragfähigen Antworten auf aktuelle Herausforderungen als vielfältiger Lern- und Übersetzungsprozess erfolgt, könnte sie zu einem echten Modell für sozialen Fortschritt werden. Die gegenwärtige gesellschaftliche
Lage allerdings, die stark von Identitätsaufladungen, Angst- und Ausgrenzungspolitiken geprägt ist (Wodak 2016), sieht die Hoffnung auf einen solchen, aus politischer, kultureller und religiöser Vielfalt generierten Fortschritt einem starken Gegenwind ausgesetzt.

\section{Literatur}

Amir-Moazami, Sch. (2016): Dämonisierung und Einverleibung: Die ,muslimische Frage in Europa. In: Castro Varela, M./Mecheril, P. (Hg.): Die Dämonisierung der Anderen. Rassismuskritik der Gegenwart. Bielefeld: transcript, 21-39.

Bair, J. (2012): Gesetzwerdungsprozess und inhaltliche Entwicklung des Islamgesetzes von 1912. SaThZ 16 (1), 22-41.

Bötticher, A./Mareš, M. (2001): Extremismus. Theorien - Konzepte - Formen. München: Oldenbourg.

Dopsch, H. (2001): Kleine Geschichte Salzburgs. Stadt und Land. Salzburg: Pustet.

Gabriel, K./Höhn, H.-J. (Hg.) (2008): Religion heute - öffentlich und politisch. Provokationen, Kontroversen, Perspektiven. Paderborn: Schöningh.

Gabriel, K./Gärtner, Ch./Pollack, D. (Hg.) (2014): Umstrittene Säkularisierung. Soziologische und historische Analysen zur Differenzierung von Religion und Politik. 2. Aufl. Berlin: Berlin University Press.

Gmainer-Pranzl, F. (2015): Religionswissenschaft: ein interdisziplinärer und interkultureller Diskurs im Spannungsfeld von Gerechtigkeit, Erkenntnis und Spiritualität. In: Fornet-Betancourt, R. (Hg.): Bildungstraditionen, Spiritualität und Universität. Perspektiven zur interkulturellen Transformation akademischer Ausbildung. Dokumentation des XI. Internationalen Kongresses für Interkulturelle Philosophie (Denktraditionen im Dialog: Studien zur Befreiung und Interkulturalität, 40). Aachen: Verlag Mainz, 127-145.

Gmainer-Pranzl, F. (2017): Die „Wahrheit“ von Religion(en) und die „Freiheit“ der Gesellschaft. Eine systematischtheologische Analyse. In: Strohmeyer, A./Oetzel, L. (Hg.): Historische und systematische Fallstudien in Religion und Politik vom Mittelalter bis ins 21. Jahrhundert (SID 9). Frankfurt/M.: Peter Lang, 23-48.

Goujon, A./Jurasszovich, S./Potančoková, M. (2017): Demographie und Religion in Österreich. Szenarien 2016 bis 2046. Deutsche Zusammenfassung und englischer Gesamtbericht (Hg. Österreichischer Integrationsfonds). Wien.

Habermas, J. (2005a): Religion in der Öffentlichkeit. Kognitive Voraussetzungen für den „öffentlichen Vernunftgebrauch“ religiöser und säkularer Bürger. In: Habermas, J.: Zwischen Naturalismus und Religion. Philosophische Aufsätze. Frankfurt/M.: Suhrkamp, 119-154.

Habermas, J. (2005b): Vorpolitische Grundlagen des demokratischen Rechtsstaates? In: Habermas, J.: Zwischen 
Naturalismus und Religion. Philosophische Aufsätze. Frankfurt/M.: Suhrkamp, 106-118.

Heinzmann, R. (2010): Religiöse und weltanschauliche Voraussetzungen der Menschenrechte. In: Nawrath, T./ Hildmann, P. W. (Hg.): Interkultureller Dialog und Menschenrechte (Studien zur Interkulturellen Philosophie, 20). Nordhausen: Traugott Bautz, 83-93.

Heuberger, V. (2012): Muslime in Österreich-Ungarn um 1912. SaThZ 16 (1), 5-21.

Hierl, K. (2012): Die Islamisierung der deutschen Integrationsdebatte. Zur Konstruktion kultureller Identitäten, Differenzen und Grenzziehungen im postkolonialen Diskurs (Politik, Gemeinschaft und Gesellschaft in einer globalisierten Welt, 13). Berlin: LIT Verlag.

Höhn, H.-J. (2007): Postsäkular. Gesellschaft im Umbruch Religion im Wandel. Paderborn: Schöningh.

Höhn, H.-J. (2015): Gewinnwarnung. Religion - nach ihrer Wiederkehr. Paderborn: Ferdinand Schöningh.

Jäggle, M. (2017): Austria first! Südwind Magazin. Internationale Politik, Kultur und Entwicklung 10/2017, 3.

Jagschitz, G. (1983): Der österreichische Ständestaat 19341938. In: Weinzierl, E./Skalnik, K. (Hg.): Österreich 19181938. Geschichte der Ersten Republik, Band 1. Graz: Styria, 497-515.

Johnston, W. M. (1974): Österreichische Kultur- und Geistesgeschichte. Gesellschaft und Ideen im Donauraum 1848 bis 1938 (Forschungen zur Geschichte des Donauraumes, 1). Graz: Böhlau.

Keil, M. (2015): Jüdische Geschichte Mitteleuropas von 1500 bis zur Gegenwart. Grundlagen - Entwicklungen - Brüche. ThPQ 163, 277-289.

Mattes, A. (2017): Zusammenleben als Glaubensfrage? Probleme und Überwindungsperspektiven eines integrationspolitischen Fokus auf Religion. In: Karasz, L. (Hg.): Migration und die Macht der Forschung. Kritische Wissenschaft in der Migrationsgesellschaft, Wien: ÖGB Verlag, 193-208.

Metz, J. B. (1977): Glaube in Geschichte und Gesellschaft. Mainz: Matthias Grünewald.

Moussaoui, A. S. (2002): Zacarias Moussaoui, mein Bruder. Zürich: Pendo.

Münz, Ch. (1996): Der Welt ein Gedächtnis geben. Geschichtstheologisches Denken im Judentum nach Auschwitz, 2. Aufl. Gütersloh: Chr. Kaiser Gütersloher Verlagshaus.

Nussbaum, M. C. (2016): Nicht für den Profit! Warum Demokratie Bildung braucht. 2. Aufl. Mühlheim an der Ruhr: TibiaPress.

Österreichische Bischofskonferenz (1952): Hirtenbriefe. Das „Mariazeller Manifest von 1952. Online: www.bischofskonferenz.at/hirtenbriefe/das-mariazeller-manifestvon-1952 [25.08.2017].

Renusch, A. (2014): Der eigene Glaube und der Glaube der anderen. Philosophische Herausforderungen religiöser Vielfalt (Thesen, 59). Freiburg/München: Alber Thesen.
Schmidt-Leukel, P. (2005): Gott ohne Grenzen. Eine christliche und pluralistische Theologie der Religionen. Gütersloh: Gütersloher Verlagshaus.

Siebenrock, R. (2013): „Eine freie Kirche in einer freien Gesellschaft": Kirche und politische Gemeinschaft. Zum politischen Handeln der „,römisch“-katholischen Kirche in Geschichte und Gegenwart. In: Nautz, J./Stöckl, K./ Siebenrock, R. (Hg.): Öffentliche Religionen in Österreich. Politikverständnis und zivilgesellschaftliches Engagement (Edition Weltordnung - Religion - Gewalt, 12). Innsbruck: innsbruck university press, 69-90.

Tálos, E. (2013): Das austrofaschistische Herrschaftssystem. Österreich 1933-1938 (Politik und Zeitgeschichte, 8), 2. Aufl. Wien/Berlin: LIT Verlag.

Vocelka, K. (2013): Multikonfessionelles Österreich. Religionen in Geschichte und Gegenwart. Wien: styria premium.

Weigl, A. (2008): Katholische Bastionen. Die konfessionellen Verhältnisse vom Vorabend des Ersten Weltkriegs bis in die frühen 1920er-Jahre. In: Konrad, H./Maderthaner, W. (Hg.): Das Werden der Ersten Republik. ... der Rest ist Österreich, Band I. Wien: CGS, 381-392.

Weinzierl, E. (1983): Kirche und Politik. In: Weinzierl, E./ Skalnik, K. (Hg.): Österreich 1918-1938. Geschichte der Ersten Republik, Band 1. Graz: Styria, 437-496.

Weinzierl, E. (1995): Der Februar 1934 und die Folgen für Österreich (Wiener Vorlesungen im Rathaus, 32). Wien: Picus Verlag.

Willems, U./Pollack, D./Basu, H./Gutmann, T./Spohn, U. (Hg.) (2013): Moderne und Religion. Kontroversen um Modernität und Säkularisierung. Bielefeld: transcript.

Wimmer, F. M. (2004): Interkulturelle Philosophie. Eine Einführung (UTB 2470). Wien: Facultas.

Wodak, R. (2016): Politik mit der Angst. Zur Wirkung rechtspopulistischer Diskurse. Wien/Hamburg: Edition Konturen.

Zulehner, P. M. (2005): Religion in Austria. In: Bischof, G./ Pelinka, A./Denz, H. (Hg.): Religion in Austria (Contemporary Austrian Studies, 13). Innsbruck: Studienverlag, $37-62$.

Zulehner, P. M./Polak, R. (2006): Religion - Kirche - Spiritualität in Österreich nach 1945. Befund, Kritik, Perspektive (Österreich - Zweite Republik. Befund, Kritik, Perspektive, 13). Innsbruck: Studienverlag. 